\title{
A dissecting aneurysm of the sinus of Valsalva involving the interventricular septum in a patient with syphilis and a quadricuspid aortic valve
}

\author{
Wenjuan Bai ${ }^{1}$, Xiang $\mathrm{Li}^{1}$, Yue Zhong ${ }^{1}$, and Li Rao ${ }^{1}$ \\ ${ }^{1}$ West China hospital of Sichuan University
}

January 7, 2021

\begin{abstract}
Aneurysms of the sinus of Valsalva are rare, with dissecting aneurysms of the sinus of Valsalva that extend into the interventricular septum being even more rare. This report describes a young patient with syphilis and a quadricuspid aortic valve who experienced a spontaneously dissecting aneurysm of the sinus of Valsalva and the basal interventricular septum.
\end{abstract}

\section{A dissecting aneurysm of the sinus of Valsalva involving the interventricular septum in a patient with syphilis and a quadricuspid aortic valve}

Wenjuan Bai ${ }^{1}$, MD, PhD Xiang $\mathrm{Li}^{2}$, MD Yue Zhong ${ }^{1}$, MD Li Rao ${ }^{1}$, MD, PhD

1 Cardiology Department, West China Hospital, Sichuan University, China

2 Cardiac Surgery Department, West China Hospital, Sichuan University, China

Short title: Dissecting aneurysm of the sinus of Valsalva

Corresponding author

Wenjuan Bai, MD, PhD

Department of Cardiology, West China Hospital of Sichuan University

NO. 37, Guoxue Street, Wuhou District, Chengdu 610041, China

Tel: 00862885422355 Mobile: +8618980606470 Email: littlewhitehx@126.com 27484197@qq.com

\section{Acknowledgements:}

This study was supported by the grant from 1.3.5 project for disciplines of excellence-Clinical Research Incubation Project, West China Hospital, Sichuan University. NO. 2020HXFH044. The funders had no role in study design, data collection and analysis, decision to publish, or preparation of the manuscript.

\section{Abstract}

Aneurysms of the sinus of Valsalva are rare, with dissecting aneurysms of the sinus of Valsalva that extend into the interventricular septum being even more rare. This report describes a young patient with syphilis and a quadricuspid aortic valve who experienced a spontaneously dissecting aneurysm of the sinus of Valsalva and the basal interventricular septum.

Keywords Dissecting aneurysm, Sinus of Valsalva, Interventricular septum, Tertiary syphilis 
A 27-year-old man presented to the emergency room after 3 days of chest and abdominal pain and vomiting. Six months earlier, he began experiencing occasional chest pains and became fatigued, with progressive worsening in the previous 2 months. One month before presentation, he was admitted to another hospital and diagnosed with pleural effusion and syphilis. Chest drainage was successful, and the patient recovered. After discharge, he was prescribed benzathine penicillin $\mathrm{G}$ infusion for 20 days. Initial physical examination in the emergency room of our hospital showed that his blood pressure was $116 / 38 \mathrm{mmHg}$, his pulse was 80 beats/min with arrhythmia, and he showed significant cardiac enlargement, although no murmur was heard in the five valve auscultation areas. His serum brain natriuretic peptide concentration was $4950 \mathrm{ng} / \mathrm{L}$ (reference range: 0-88 ng/L) and his troponin concentration was slightly elevated. Screening for syphilis showed a 1:16 titer on a toluidine red unheated serum test and a positive result on a T. pallidum passive particle agglutination assay. Electrocardiography revealed a premature ventricular beat.

Transthoracic echocardiography showed a large aneurysm of the sinus of Valsalva located on the left side of the aortic root and close to the right ventricle. The aneurysm wall dissected and extended into the basal segment of the interventricular septum (Figure A and B, movie clip S1). Color Doppler flow imaging showed low-speed shunting through the perforation from the sinus of Valsalva into the dissecting aneurysm (Figure C). Three-dimensional echocardiography image showed a intimal tear in the aortic root (Figure D). In addition, a quadricuspid aortic valve was observed (movie clip S2). There was severe aortic regurgitation, and no vegetation was observed on the aortic valves. Contrast-enhanced computed tomography $(\mathrm{CT})$ confirmed a giant aneurysm of the left sinus of Valsalva measuring $9.5 \times 7.5 \mathrm{~cm}$ (Figure E, F and G).

Subsequent open heart surgery confirmed the dissecting aneurysm of the sinus of Valsalva and the involvement of the basal interventricular septum. Aortic wall thickening with calcification and a quadricuspid aortic valve were coincidentally observed. The aortic valves were restricted and insufficient. The aortic valve, the aortic root, and the ascending aorta were replaced by a mechanical valve and artificial graft, and the right coronary artery was implanted into the artificial graft. Intraoperative transesophageal echocardiography showed that the mechanical aortic valve worked well. The patient's postoperative course was uneventful and he was discharged in good condition. Histopathologic analysis of the aorta revealed focal thickening of the arterial intima with fibrous tissue hyperplasia, multiple focal destruction of the medial structure with chronic infiltration of inflammatory cells, and scattered lymphoid follicles in the peripheral membrane with small vascular hyperplasia (Figures H and I). These results were consistent with meso-aortitis syphilitica. Follow-up after 3 months showed that the patient was asymptomatic.

\section{Discussion}

The incidence of cardiovascular syphilis, which was high during the first half of the 20th century due to the lack of effective treatment, decreased significantly after the introduction of penicillin to treat syphilis ${ }^{1-3}$. Since the beginning of the 21st century, however, the incidence of syphilis has increased annually. ${ }^{4}$ Tertiary syphilis is less common today, perhaps owing to the widespread use of antibiotics. ${ }^{5,}{ }^{6}$ Cardiovascular syphilis is a late complication of untreated tertiary syphilis, affecting an estimated $7.4 \%$ of these patients. ${ }^{5}$ Most patients with cardiovascular syphilis have aortic insufficiency, aortic aneurysm, and/or coronary ostial stenosis. ${ }^{7-8}$ However, dissecting aneurysms of the sinus of Valsalva are very rare.

A quadricuspid aortic valve is an uncommon congenital valve lesion, and the incidence of aortic regurgitation increases with age in patients with this lesion; however, surgery can be used to replace the aortic valve until the fifth or sixth decade of life..$^{9}$ The patient described in the current report was young and had not been previously treated for syphilis or an aortic valve defect. The combination of syphilis with a quadricuspid aortic valve may have resulted in the dissecting aneurysm of the sinus of Valsalva. In our report, echocardiography and cardiac CT provided high quality anatomic detail for precise diagnosis. Multiformat reconstructions and volume rendered techniques were useful for pre-operative planning.

\section{Figure legends}

Figure Multimodal imaging and pathological findings. 
A and B Transthoracic echocardiography showing a dissecting aneurysm of the sinus of Valsalva involving the interventricular septum.C The two-dimensional and color Doppler comparative focused images of the parasternal long-axis view of the aortic root show low-speed shunting through the ruptured flow into the aneurysm (white arrows). D Three dimensional echocardiography image viewed from the aortic root, showing intimal tears in the sinus (dotted line).E Computed tomography angiography showing a large aneurysm arising from the sinus of Valsalva. F and G Light microscopy examination of the aortic aneurysm showing focal thickening of the arterial intima with fibrous tissue hyperplasia and multiple focal destruction of the medial structure of the artery with chronic infiltration of inflammatory cells, primarily plasma cells ( $\mathrm{H} \& \mathrm{E}$ $\times 25, \times 200$, respectively). LV, left ventricle; LA, left atrium; RV, right ventricle; RA, right atrium; AV, aortic valve; Ao, aorta; IVS, interventricular septum; PA, pulmonary artery; Aneu, aneurysm.

Movie clip S1 . Transthoracic echocardiography shows a dissecting aneurysm of the sinus of Valsalva involving the interventricular septum.

Movie clip S2 . The short axis view shows the quadricuspid aortic valve.

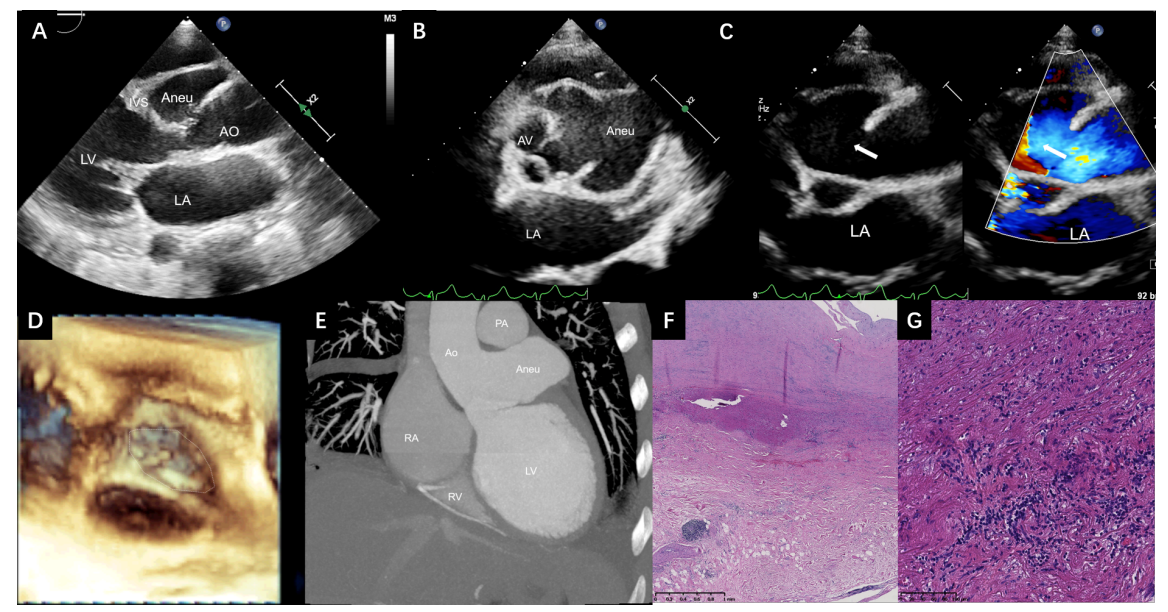

